von der wässerigen Phase getrennt werden. - Wird zur Fällung der Eiweiße lediglich Äthanol verwendet, so ist dieser Nachteil ausgeschaltet. Verluste an Gallensäuren treten ferner auf bei der anschließenden Extraktion in einem unpolaren organischen Lösungsmittel. Dieser Arbeitsgang ist in der hier beschriebenen Methode durch die präparative dünnschichtchromatographische Vorreinigung der äthanolischen Lösung ersetzt.

In der vorliegenden Methodik werden die Testsubstanzen einem Mischserum zugesetzt und durch den gesamten Analysengang mitgeführt (Tab. 1). Dies hat den Vorteil, daß die Verluste während des Arbeitsprozesses bei der Berechnung der Konzentration der Gallensäuren in den Seren (Tab. 2) von vornherein miteinbezogen sind. - Mit der Methode können Werte von über $0,1 \mathrm{mg} \%$ semiquantitativ bestimmt werden.
Die Werte für die konjugierten Gallensäuren liegen in mehr als der Hälfte der Bestimmungen unter der Nachweisgrenze von $0,1 \mathrm{mg} \%$. Damit ist eine weitgehende Ubereinstimmung mit den von Frosch $(6,7)$ mitgeteilten Werten erreicht. Bei den freien Gallensäuren, vor allem der Cholsäure, wurden in der vorliegenden Arbeit im Vergleich zu Frosch etwas höhere Konzentrationen nachgewiesen. Die Werte für die freie Cholsäure liegen in den hier untersuchten Fällen deutlich über denjenigen der beiden Dihydroxysäuren (DC $+\mathrm{CDC}$ ) sowie der konjugierten Gallensäuren.

Ich danke der Firma Merck Sharp und Dohme, Rahway (New Jersey) und den Herren Dr. U. P. Hämmerl.I und Dr. J. Müller von der Medizinischen Universitätsklinik Zürich, durch deren großzügiges Entgegenkommen die vorliegende Arbeit ermöglicht wurde.

\title{
Literatur
}

1. Josephon, B., Biochem. J. 29, 1519 (1935). - 2. Kier, L. C., J.Laborat. Clin. Med., S. Louis, 40, 755 (1952). - 3. Levin, S. J. und $C_{\text {H. }}$ G. Johnston, J. Laborat. Clin. Med., S. Louis, 59, 681 (1962). - 4. Lirvin, J., C. G. Johnston und J. Kopala, J. biol. Chemistry 153, 439 (1944). - 5. MrNiBECK, H., Biochem. Z. 297, 29, 40 und 214 (1938). - 6. Frosch, B., Arzneimittel Forsch. 15, 178 (1965). - 7. Frosch, B., Klin. Wschr. 43, 262 (1965). - 8. Tsuyoshr, I. und K. Yamasak,; J. Biochemistry (Tokyo) 56, 5
(1964). - 9. Sandberg, D., K. Sjövall und D. A. Turner, Federat. Proc. 22, 198 (1963). - 10. Patakr, G., Dünnschichtchramotographie in der Aminosäure- und Peptidchemie. (Im Druck), De Gruyter u. Co., Berlin. - 11. BrenNer, M., A. Niederwieser, G. Pataki und A. R. Fahmy, Experientia (Basel) 18, 101 (1962). - 12. Bergström, S. und A. Norman, Acta chem. scand. 7,1126 (1953).

\section{Zur Abhängigkeit der Bestimmung der ,wahren Glucose“ von der Glas-Sorte der Reagenzgläser ${ }^{1)}$}

\author{
Von W. Forth und K. Pfleger \\ Aus dem Pharmakologischen Institut der Universität des Saarlandes, Homburg/Saar (Direktor: Prof. Dr. W. Rum mel)
}

(Eingegangen am 11. Mai 1965)

\begin{abstract}
Eine systematische Prüfung der von Frank und Kirberger modifizierten Methode zur Bestimmung der ,wahren Glucose" nach NeLson zeigt, daß es nicht gleichgültig ist, in welcher Glas-Sorte die Oxydation der zu messenden Glucose bzw. die stöchiometrische Reduktion des Kupfers stattfindet. Bei der Bestimmung von Glucose in Lösungen, deren Konzentration 30-60 mg\% betrug, werden in Fiolax-Gläsern z. B. nur 20-30\% der Einwaage erfaßt. In Duran- bzw. Pyrex-Glas macht sich diese Störung nicht bemerkbar. Wahrscheinlich ist die Störung auf den Arsen-Gehalt des Fiolax-Glases zurückzuführen.

The systematical control of the method of NeLson for the estimation of glucose modified by Frank. and KIrberger showed, that it is not indifferent in which kind of glass the oxidation of glucose or the stechiometrical reduction of copper is carried out. The determination of glucose in a concentration of $30-60 \mathrm{mg} \%$ in Fiolax test tubes yields only $20-30 \%$ of the total amount whereas in Duran i. e. Pyrex test tubes a $100 \%$ yield is achieved. It is most likely that the interference of Fiolax glass with the method depends on arsenic found in this glass.
\end{abstract}

Nach Einführung einer neuen Reagenzglas-Sorte in unserem Institut (1960), in dem bis dahin ausschließlich Pyrex-Glas französischer Herkunft verwendet wurde, machten häufiger auftretende Störungen die Überprüfung der von Frank und KIRBERGER modifizierten Methode zur Glucose-Bestimmung nach NELSON nötig

1) Uber einen Teil der Ergebnisse wurde auf der gemeinsamen Tagung der Deutschen Gesellschaft für Physiologische Chemie und der Osterreichischen Biochemischen Gesellschaft in Wien (26.-29. 9. 1962) berichtet.
(2). Dabei fiel auf, daß regelmäßig in bestimmten Reagenzgläsern aus Fiolax-Glas die Werte der GlucoseBestimmung in ein und derselben Lösung erheblich niedriger ausfielen als in Pyrex-Gläsern, die wir mit den neuen Reagenzgläsern zunächst wahllos vermischt benutzten. Die Störungen der Glucose-Bestimmung in Fiolax-Glas waren besonders bei der Messung niedriger Glucose-Konzentrationen ausgeprägt, d. h. in einem Konzentrations-Bereich, der - auf die Blutzuckerbestimmung übertragen - klinisch gerade besonders 
interessant ist. Im Folgenden wird über die Untersuchung der Ursachen dieser Störung berichtet.

\section{Methode}

Der Glucose-Bestimmung nach NeLson in der Modifikation von FraNk und KIRBERger liegt folgendes Prinzip zu Grunde:

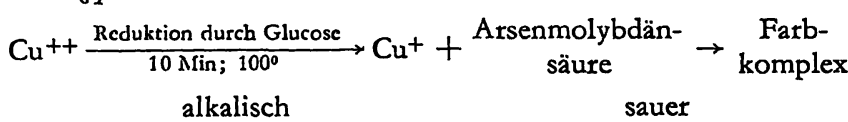

Wie eine Reihe anderer Glucose-Bestimmungen beruht auch diese Methode auf der Tatsache, daß $\mathrm{Cu}^{2+}$ in alkalischem Milieu durch Glucose reduziert wird. $\mathrm{Cu}$ wird als $\mathrm{Cu}(\mathrm{II}) \mathrm{SO}_{4}$ einem BikarbonatPuffer von $\mathrm{pH} 10$ zugesetzt. Mit dieser Lösung wird die enteiweißte. Analysen-Probe versetzt und 10 Minuten im Wasserbad gekocht. Dabei bildet sich durch Reduktion des $\mathrm{Cu}^{2+}$ durch die Glucose $\mathrm{Cu}(\mathrm{I}) \mathrm{OH}$. In der zweiten Reaktionsfolge wird dem Analysenansatz Arsenmolybdänsäure zugesetzt, wobei in saurem Milieu durch Reduktion des Molybdates ein blauer Farbkomplex entsteht, dessen Konzentration photometrisch bestimmt wird.

Die Methode wurde genau nach Vorschrift, wie sie in der Anleitung für photometrische Bestimmungen mit dem Photometer „Eppendorf“ von der Firma Netheler und Hinz (Hamburg) herausgegeben wurde, durchgeführt. Wir haben mit Reagenzgläsern aus qwei GlasSorten gearbeitet: 1. Duran-Glas der Firma Schott \& Gen. (Mainz); dieses Glas ist mit Pyrex-Glas anderer Firmen identisch. 2. Fiolax-Glas der Firma Schott und Gen. (Mainz). Die Glucose-Bestimmungen wurden in Tyrode-Lösung folgender Zusammensetzung durchgeführt: $8,0 \mathrm{NaCl} ; 0,2 \mathrm{KCl} ; 0,2 \mathrm{CaCl}_{2} \cdot 6 \mathrm{H}_{2} \mathrm{O} ; 0,1$ $\mathrm{MgCl}_{2} \cdot 6 \mathrm{H}_{2} \mathrm{O} ; 1,0 \quad \mathrm{NaHCO}_{3} ; 0,05 \mathrm{NaH}_{2} \mathrm{PO}_{4}$. Die Glucose-Konzentration wurde, wie im folgenden angegeben, variiert.

\section{Ergebnisse}

In Tabelle 1 sind die Ergebnisse der Glucose-Bestimmung in Duran-Gläsern und Fiolax-Gläsern in Lösungen mit steigenden Glucose-Konzentrationen zusammengefaßt.

Tab. 1

Glucose-Bestimmung nach NeLson (modif. von FranK und KIRBERGER) in Duran-und Fiolax-Reagenzgläsern

(Werte in $\mathrm{mg} \%$ )

\begin{tabular}{rrrrr}
\hline & & \multicolumn{4}{c}{ Gefunden in: } \\
Einwaage & n & Duran-Glas & \multicolumn{1}{c}{ Fiolax-Glas } \\
\hline 30,4 & 23 & $27,1 \pm 5,2$ & $6,3 \pm 8,8$ \\
60,8 & 18 & $57,0 \pm 4,3$ & $16,5 \pm 21,6$ \\
152,1 & 14 & $146,2 \pm 11,2$ & $106,1 \pm 41,0$ \\
304,0 & 15 & $313,6 \pm 8,1$ & $292,5 \pm 61,5$ \\
\hline
\end{tabular}

Es fällt auf, daß in einem Bereich von $30-150 \mathrm{mg} \%$, der klinisch besonders interessiert, in Fiolax-Gläsern Minderbestimmungen um $60-80 \%$ auf die Einwaage bezogen erhalten wurden. Daneben ist die Streuung bei Fiolax-Gläsern viel größer als bei Duran-Gläsern; sie beträgt beispielsweise für die Einwaage $152,1 \mathrm{mg} \%$ in Duran-Glas $8 \%$ des gemessenen Mittelwertes, für
Fiolax-Glas dagegen 39\%. Erst bei Glucose-Konzentrationen von $300 \mathrm{mg} \%$ unterscheiden sich die Mittelwerte beider Glas-Sorten nicht mehr. Die Streuung in der Gruppe der Fiolax-Gläser beträgt àber immer noch $20 \%$ des Mittelwertes, die der in Duran-Gläsern gemessenen Werte nur $2 \%$.

Bei den Untersuchungen fiel auf, daß nach dem Kochvorgang, nach dem normalerweise die Bildung des schwerlöslichen $\mathrm{CuOH}$ mit dem bloßen Auge beobachtet werden kann, in Fiolax-Gläsern bei geringen GlucoseKonzentrationen die gelbliche bis rötliche Farbe des $\mathrm{CuOH}$ ausblieb. Dies deutete bereits darauf hin, daß die Störung der Methode möglicherweise in dem Abschnitt liegt, in dem $\mathrm{Cu}^{2+}$ durch Glucose $\mathrm{zuCu}+$ reduziert wird. Zur Klärung der Frage, in welchem Teil des Analysengangs die Störung liegt, ließen wir nach Reduktion des Kupfers in Duran-Glas anșchließend die Bildung des Farbkomplexes zum Teil in Fiolax-Glas und zum Teil in Duran-Glas ablaufen.

Aus Tabelle 2 geht hervor, daß bei diesem Vorgehen kein Unterschied zwischen den so gewonnenen Ergebnissen zu beobachten ist. Demnach ist die Störung bei der Reduktion des Kupfers durch die Glucose zu suchen.

Tab. 2

Glucose-Bestimmung nach NeLsoN (modif. von FranK und KIrberger); nach Reduktion des Kupfers in Duran-Gläsern Bildung des Farbkomplexes in Duran- bzw. Fiolax-Gläsern. (Werte in $\mathrm{mg} \%$ )

Einwage: $39,9 \mathrm{mg} \% ; \mathrm{n}=7$ Gefunden in:

$\begin{array}{cc}\text { Duran-Glas } & \text { Fiolax-Glas } \\ 39,6 \pm 2,3 & 41,7 \pm 3,0\end{array}$

Es konnte ausgeschlossen werden, daß die zu bestimmende Glucose etwa durch Veränderungen an ihrer Aldehyd-Gruppe der Bestimmung entzogen wird. Nach Kochen kleiner Glucose-Mengen in alkalischem Milieu und in Fiolax-Gläsern konnte die Glucose ohne weiteres enzymatisch bestimmt werden (Glucose-Oxydase-Methode). - $\mathrm{Da}$ die meisten Labor-Glas-Sorten einen beachtlichen Gehalt an Bor aufweisen (7-13\%) (4), war auch daran zu denken, daß die Glucose wie andere Polyhydroxy-Verbindungen (1) mit dem Borat des Glases einen Komplex bildet, der dann u. Ũ. die Glucose der Bestimmung entziehen könnte. Die Bor-Konzentrationen, die eine merkliche Beeinträchtigung der GlucoseBestimmung verursachen, liegen jedoch sehr hoch: es müßten 300-400 $\mu \mathrm{g}$ Bor/Ansatz vorliegen. Die funkenspektrographische Untersuchung des alkalischen Kochsaftes aus beiden Glas-Sorten ergab überdies, daß DuranGlas mehr Bor in Lösung abgibt, als Fiolax-Glas, das auch gegen Alkalien korrosionsbeständiger ist als Duran-Glas (6).

Schon Frank und KIRberger (2) wiesen mit Nachdruck auf die Störungen hin, die sie bei der Ausarbeitung ihrer Methode in Gläsern beobachteten, die mit Bichromat gespült wurden. Wir vermieden daher von vornherein 
diese Art der Gläser-Reinigung. Jede Art Spülung, ob reine Wasser-Spülung oder Spülung mit konz. $\mathrm{HCl}$ oder $\mathrm{HNO}_{3}$, blieb ohne Einfluß auf die Störung der GlucoseBestimmung in Fiolax-Gläsern.

Es galt nun zu prüfen, ob Fiolax-Glas beim Kochvorgang während der Glucose-Bestimmung, der in alkalischem Milieu ( $\mathrm{pH} \mathrm{10)} \mathrm{stattfindet,} \mathrm{ein} \mathrm{störendes} \mathrm{Agens}$ in die Lösung abgibt. Zu diesem Zweck schmolzen wir in Fiolax- und Duran-Reagenzgläser einige $\mathrm{m} l$ Bikarbonat-Puffer ein und kochten diese Lösung eine Stunde im Wasserbad. Mit dem so gewonnenen Kochsaft der Gläser stellten wir nach dem Erkalten regelrechtes Glucose-Reagenz her. Wir führten die Glucose-Bestimmung in Duran-Gläsern mit Reagenz aus Fiolax-GlasKochsaft durch, nachdem zuvor geprüft wurde, daß das Reagenz, das aus Duran-Glas-Kochsaft hergestellt wurde, funktionstüchtig war. Dabei zeigte sich nun überraschenderweise, daß die Glucose-Bestimmung in Duran-Glas unter Verwendung von Fiolax-Glas-Kochsaft ohne Störung durchgeführt werden konnte. Aufgrund dieser Ergebnisse muß in Betracht gezogen werden, daß das die Glucose-Bestimmung störende Agens des Fiolax-Glases gar nicht aus dem Glas herausgelöst werden kann.

Nachdem nun festzustehen schien, daß einerseits kein störender Inhaltsstoff des Fiolax-Glases bei der GlucoseBestimmung herausgelöst wird, andererseits aber die Störung der Glucose-Bestimmung offensichtlich durch den Kontakt des Bestimmungsansatzes mit dem FiolaxGlas zustande kommt, ließen wir die Bestimmung in Fiolax-Reagenzgläsern ablaufen, deren Oberfläche silikoniert war. In diesen Gläsern, deren Oberfläche keinen direkten Kontakt mit der Lösung des Bestimmungsansatzes hatte, unterschieden sich die Glucose-Werte nicht von denen, die in Duran-Gläsern gemessen wurden. Bei diesem Sachverhalt lag es nahe, an feste Ionen in der Oberfläche der Glasstruktur als Wirkungsträger $\mathrm{zu}$ denken, die möglicherweise durch ihre oxydationskatalytischen Eigenschaften die Glucose-Bestimmung stören können.

Auf der Suche nach Inhaltsstoffen des Fiolax-Glases, die zur Erklärung der beobachteten Störung der GlucoseBestimmung dienen könnten, bedienten wir uns der funkenspektrographischen Untersuchung von FiolaxGlasgries, der mit dem Gries aus Duran-Glas verglichen wurde. Dabei zeigte sich, daß Fiolax-Glas im Unterschied zum Duran-Glas Arsen enthält. Qualitativ scheinen sich Fiolax- und Duran-Glas sonst nicht zu unterscheiden. In der Folge wurde uns auch bestätigt, daß Fiolax-Glas während der Schmelze einen $\mathrm{As}_{2} \mathrm{O}_{3}$-Zusatz erhält, der in der fertigen Röhre 0,08\% ausmacht (5). - Z War läßt sich durch Zusatz eines Stoffes zum Analysen-Ansatz nicht ohne weiteres auf sein Verhalten als Fest-Ion an der Oberfläche der Glasstruktur schließen, jedoch konnte durch Zusatz von $\mathrm{As}_{2} \mathrm{O}_{3}$ zum Versuchsansatz vor dem Kochvorgang wahrscheinlich gemacht werden, daß As als Störfaktor durchaus in Frage kommt. Bereits ein Zusatz von $1,5 \mu \mathrm{g} \mathrm{As} / \mathrm{ml}$ bewirkte eine Verminderung der gemessenen Glucose-Konzentration um 57\%. Von der eingewogenen Glucose-Konzentration von $29 \mathrm{mg} \%$ wurden als Mittelwert von 5 Einzelmessungen $12,4 \mathrm{mg} \% \pm 6,7$ bestimmt.

Wir haben es bei diesem Stand der Untersuchungen, die aus einer notwendig gewordenen Überprüfung einer bisher bewährten Methode resultierten, bewenden lassen, nachdem es wahrscheinlich schien, daß der ArsenGehalt des Fiolax-Glases die Ursache der Störung ist.

Uber den Mechanismus der Störung der Reduktion des $\mathrm{Cu}$ durch As bei der Glucose-Bestimmung können wir nur Vermutungen anstellen. Es ist bekannt, daß As mit seinen verschiedenen Wertigkeitsstufen ein Oxydoreduktionspotential besitzt (3). Theoretisch kann man sich vorstellen, daß entweder As gegenüber $\mathrm{Cu}$ bei der Reduktion durch Glucose bevorzugt wird, oder aber daß durch Glucose reduziertes $\mathrm{Cu}$ durch das As-System wieder oxydiert wird. In beiden Fällen resultiert aus der Anwesenheit von As im Versuchsansatz eine Verminderung des zum Glucose-Gehalt der Probe stöchiometrisch reduzierten $\mathrm{Cu}$.

Die Frage nach dem der Störung der Glucose-Bestimmung zu Grunde liegenden Mechanismus ist weniger der Sinn dieser Mitteilung, als vielmehr der Hinweis darauf, daß Glas, ein gewöhnlich inert erscheinender Werkstoff, vielleicht öfter Anlaß zu Störungen chemischer Methoden ist, als man bisher ahnt. Vielleicht ist dieser Bericht auch Anlaß dafür, die Prüfungskriterien zur Beurteilung chemischer Eigenschaften von Glas, die sich bislang nur auf die Resistenz gegen Wasser, allenfalls Säuren und Laugen erstrecken $(4,6)$, neu $\mathrm{zu}$ überdenken.

Herrn Prof. Dr. H.-J. Staudinger, Gießen und Herrn Prof. Dr. H.-J. Bielig, Saarbrücken danken wir herzlich für die anregenden Diskussionen und Frau H. WILBERT, Homburg für ihre unermüdliche technische Mitarbeit.

\section{Literatur}

1. Bersin, TH., Biochemie der Mineral-und Spurenelemente, S. 518. Akademische Verlagsgesellsch., Frankfurt Main (1963). - 2. Frank, H. und E. Kirberger, Biochem. Z. 320, 359 (1950). 3. HOLLDORF, A.W., Gleichgewichtskonstanten, freie Energie und Redoxpotentiale biologisch wichtiger Reaktionen, in: Biochem. Taschenbuch II. S. 142 Hrsgb. H. M. RaUEN. Springer-
Verlag, Berlin-Göttingen-Heidelberg-New York (1964). - 4. ReHM, K., Österr. Chemiker-Ztg. 63, 10 (1962). - 5. ReHM, K., persönl. Mitteilung (1963). - 6. SchотT \& GEN. (Herausgeber), Physikalische und chemische Eigenschaften technischer Gläser. Jena'er Glaswerk Schott \& Gen., Mainz (1959).
Dr. W. Forth

Pharmakolog. Institut der Universität des Saarlandes 665 Homburg (Saar) 